\title{
A Comparative Study of Classification Techniques for P300 Speller
}

\author{
VaishaliPatelia, Maulika S. Patel
}

\begin{abstract}
P300 speller in Brain Computer Interface (BCI) allows locked-in or completely paralyzed patients to communicate with humans. To achieve the performance of characterization and increase accuracy, machine learning techniques are used. The study is about an event related potential (ERP) P300 signal detection and classification using various machine learning algorithms. Linear Discriminant Analysis (LDA) and Support Vector Machine (SVM) are used to classify P300 and Non-P300 signal from Electroencephalography (EEG) signal. The performance of the system is evaluated based on f1-score using BCI competition III dataset II. In our system, we used LDA and SVM classification algorithms. Both the classifiers gave $91.0 \%$ classification accuracy.
\end{abstract}

Keywords: Brain Computer Interface, P300 Speller, Event Related Potential, Linear Discriminant Analysis, Support Vector Machine.

\section{INTRODUCTION}

B rain Computer Interface (BCI) is a neural control system which translates brain activity via signals into computer or electrical devices. BCI system allows amyotrophic lateral sclerosis [2] patients to communicate with others through a brain signals. Various research fields of the BCI are medical, robotics, security, education [1] etc. Muse and Aurora [3] are the BCI application devices use for different purpose as sleeping. BCI system used Electroencephalography (EEG) signal which is a type of non-invasive method to record electrical activity of the brain recorded by headset device, evoked at a specific frequency. The EEG signals are classified in alpha, beta, gamma \& delta based on their frequency range. Event related potential (ERP) [13] signals are generated bymerging and taking mean of multiple channel signals. P300 signals are positive evoked ERP signals nearby $300 \mathrm{~ms}$. P300 speller system is the main task to classify P300 and Non-P300 signals from EEG than characterize targeted character to spell right. It may possibly that every P300 signals may not contain the character.Real time application of P300 speller is Lie Detector Machine.

BCI System, as illustrated in Fig 1 consists of following modules: signal acquisition, signal processing, classification and feedback. EEG headset device used to capture EEG signal. Signal acquisition task is to acquire signals and remove noisy data from the raw signal. Signal processing is used to translate raw signals into the specified class signals which signals are classified using classification algorithms. Now we can use targeted signal to build an application.

Revised Manuscript Received on May 20, 2020.

VaishaliPatelia, Department of Computer Engineering, G. H. Patel College of Engineering \& Technology, Gujarat Technological University, V.V. Nagar, Gujarat, India.

Maulika S. Patel, Professor \& Head, Department of Computer Engineering, G. H. Patel College of Engineering and Technology, Gujarat Technological University, V.V. Nagar, Gujarat, India.
Some of the BCI applications may use its feedback to improve result like P300 Speller.

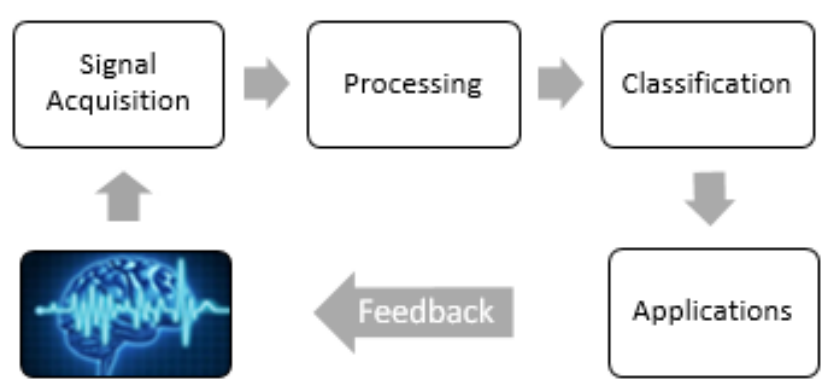

Fig 1. BCI System

BCI applications are implemented using standalone software OpenViBE [4] and BCI2000 [5, 6]. OpenViBE software is free and open-source, which is implemented in python and Matlab. OpenViBE can be used to acquire process, classify and visualize EEG signals in real time. P300 speller can be implemented in matlab and python. Both are efficient and have libraries to perform tasks. EEGLAB, BCILAB are toolbox of MATLAB. Mushu [7], Wyrm [8] and Pyff [9] are python toolbox. Mushu is an open-source EEG signal acquisition software. Wyrm is useful for offline \& online signal pre-processing and classification task. Pyff is a feedback framework which provide environment to develop BCI feedback applications. BCI competition III dataset II is used to implement speller.

\section{RELATED WORK}

Guy, Violaine, et al. [2] examined the word prediction of 20 ALS patients. This study is about three block sessions that are free spelling, copy spelling, and free usefor evaluation. The recorded calibration data is used to train spatial filter for processing and LDA classifier for classification.

Kaper, Matthias, et al. [14] proposed a method that has an ensemble of linear support vector machine classifier approach. A SVM classifier is trained on available dataset and do channel selection task. The novelty in this study is that the dataset is to split in several part and apply classifier to train the data. Output is summed of all the results of classifier.

Hongchang Shan, Yu Liu, TodorStefanov[10] proposed $\mathrm{CNN}$ architecture with only one convolution layer which is able to better learn P300-related features. Liu improves CNN by combining Batch Normalization and Dropout techniques. In One Convolution Layer Neural Network (OCLNN), they use a Rectified Linear Unit (ReLU) as an activation function to model a neuron's output because it is trained much faster than with traditional activation functions. 


\section{A Comparative Study of Classification Techniques for P300 Speller}

The Output of OCLNN are two neurons; represents the class "P300" and "non-P300" \& class "P300" used to calculate the position of the target character in the P300 speller application. This paper uses three benchmark datasets, namely, BCI Competition II - Data set IIb as well as BCI Competition III - Data set II Subject A and Subject B.

Qi, Hongzhi, et al. [11] proposed a method to improve information transfer rate and to reduce calibration time. The novel method were used a small number of ERP epochs to build a reference epoch. The Riemannian distance is used to select samples from the existing dataset, it has more subjects. Classifier are LDA, SVM, and Stepwise LDA (SWLDA) were trained on selected data, and then were used to identify the targeted character. An average Character Recognition Accuracy (CRA) achieved by classifier build on the dataset is about $90 \%$.

\section{PROCESSING}

In BCI implementation, EEG processing task aims to translate raw EEG signals into the specified class of targeted or non-targeted. Processing contain following two main steps are:

Feature Extraction: Feature extraction is used to describe the EEG signals by a relevant records called features. This feature would be contains information embedded in EEG. Extracted features are arranges in vector form, known as feature vector.

Classification: Classification aims to classify a set of feature vector to a specific class. Machine learning algorithms used for classification which are known as classifier.

\section{Feature Extraction}

Raw EEGsignals recorded using electrodes, amplifier and recording devices.Raw signals have noise of subject eye movement or minor body movement also technical noise related to cable movement, broken wire, and power fluctuation [22].Pre-processing task is to eliminate redundant features and also increase signal to noise ratio. Feature extraction used to transform row data into features suitable for modelling so that classifier are able to select corresponds input feature. Feature extraction is a dimensionality reduction process, where an initial set of row variables are reduced to more manageable group for processing.

In BCI we can extract features from EEG signals mainly 3 ways are:

Spatial information: Features are extracted from where the signals comes from. Basically, spatial information can be gathered by selecting specific EEG channels which related to specific area or portion of the brain.

Spectral (frequential) information: Such features are described by its specific frequency bands. Table 1 illustrated EEG signals frequency bands with its frequency range and how its look like.
Table 1 EEG Signal Frequency Bands

\begin{tabular}{|c|c|c|}
\hline Gamma & $30-100+\mathrm{Hz}$ & 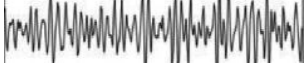 \\
\hline Beta & $14-30 \mathrm{~Hz}$ & \\
\hline Alpha & $8-13 \mathrm{~Hz}$ & \\
\hline Theta & 4-7 Hz & \\
\hline Delta & $1-3 \mathrm{~Hz}$ & \\
\hline
\end{tabular}

Temporal information: EEG signals varies with time are features comes under temporal information. In EEG signal at different time period or window values are differ.

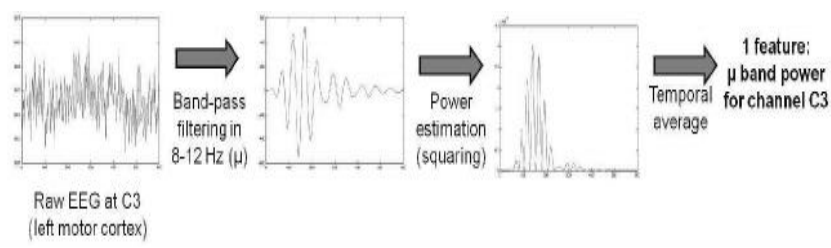

Fig 2. EEG Signal Pre-processing steps [18]

1. Sampling: The EEG signal was low pass and high pass filter and subsampled.

2. Segmentation: Define marker with segmentation interval where marker is events or cut of the data around the time interval.

3. Mean: Calculate mean of the selected channels over time intervals. Time intervals are chosen by signed $r^{2}$ values.

4. Appends: Appends the average values to generate feature vector of each trial channel. We have feature vector for each channel and each trial.

5. Now we can perform classification algorithm on generated feature vector to train model.

\section{MACHINE LEARNING ALGORITHM FOR CLASSIFICATION}

For classification purpose different algorithms are used like Linear Discriminant Analysis (LDA) [12], Support Vector Machine (SVM) [10] and Convolutional Neural Network (CNN) [14].

\section{A. Linear Discriminant Analysis}

For BCI, the most used classifier is LDA also known as Fisher's LDA. LDA is to use hyperplanes to separate the training feature vectors representing the different classes. The location and orientation of this hyperplane is determined from training data. Most probable use of LDA in $\mathrm{BCI}$ is that it has very low computational requirements, it is simple and good at generalizing unseen data also provide good result. 


\section{A Comparative Study of Classification Techniques for P300 Speller}

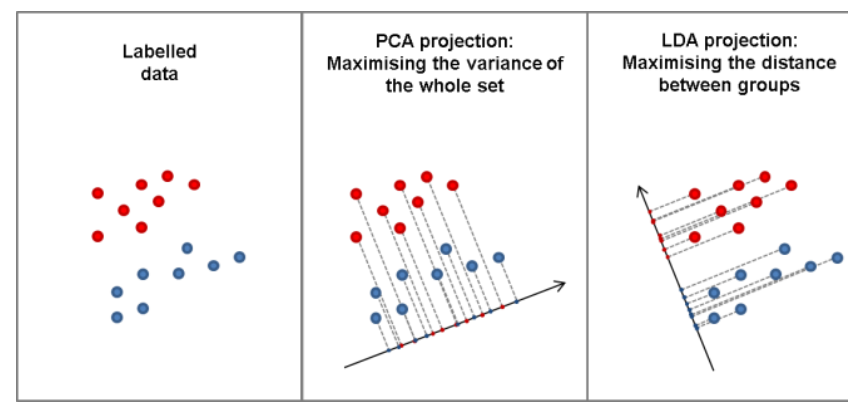

Fig 3.PCA and LDA projection lines [18]

Principal Component Analysis (PCA) [12]is the most accurate data representation for lower dimensional data but it is useless for classification purpose.LDA projection lines are maximizing the distance between groups. LDA aim at find projection to a line samples from different classes are well separated.

LDA projection minimize the class covariance of each class which result in minimum overlap.The objective is formalized as follows.

$$
J(w)=\frac{\mathrm{w}^{T} S_{b} \mathrm{w}}{\mathrm{w}^{T} S_{w} \mathrm{w}}
$$

where $S_{b} \in \mathrm{R}^{d \times d}$ and $\mathrm{S}_{\mathrm{w}} \in \mathrm{R}^{\mathrm{d} \times \mathrm{d}}$ are the between-class and within-class covariance matrices, respectively. They are calculated as

$$
\begin{aligned}
S_{b} & =\sum_{k=1}^{K}\left(m_{k}-m\right) N_{k}\left(m_{k}-m\right)^{T} \\
S_{w} & =\sum_{k=1}^{K} \sum_{n=1}^{N_{k}}\left(X_{n k}-m_{k}\right)\left(X_{n k}-m_{k}\right)^{T}
\end{aligned}
$$

Where, $X_{n k}=$ nth data example in the kth class,

$\mathrm{N}_{\mathrm{k}}=$ number of examples in class $\mathrm{k}$,

$\mathrm{m}=$ overall mean of the entire data and

$\mathrm{m}_{\mathrm{k}}=$ mean of the $\mathrm{kth}$ class.

Now using Lagrangian dual and the KKT conditions, the maximizing problem can be transformed into the solution.

$$
S_{w}^{-1} S_{b} w=\lambda_{w}
$$

Which is an eigenvalue problem for the matrix $S_{w}{ }^{-1} S_{b}$. Thus the solution for $\mathrm{w}$ will be the eigenvectors of the equation. The $\mathrm{d}^{\prime}$ largest eigenvalues will take for reduction to $\mathrm{d}^{\prime}$ dimensions. Also, note that the maximum value of $\mathrm{d}^{\prime}$ may be K-1 if we have K classes so that we can't project K class to a dimension greater than $\mathrm{K}-1$.

\section{B. Support Vector Machine}

Support Vector Machine is a supervised learning classification algorithm which classify two different classes of recorded EEG data [23]. In SVM,the optimal hyperplane is used to maximizes the distance from the nearest points and minimize distance between support vectors which is described by the vectors which lie on the margin.

SVM has advantages include insensitive to overtraining, good generalization and to the curse-of-dimensionality. SVM shows a good performance in ERP [21].

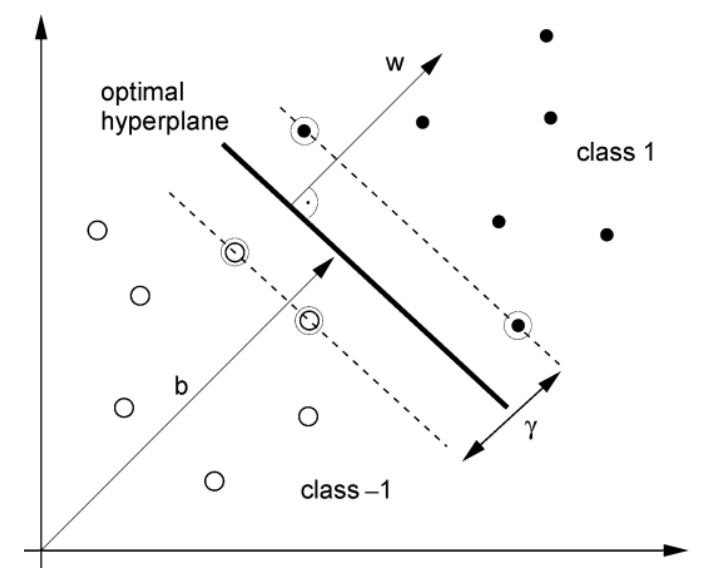

Fig 4. SVM to find the optimal hyperplane [14]

In Fig 4, optimal hyperplane is used to separate targeted and non-targeted class by maximizing margin which is described by the bias $b$ and vector w. Support vectors are bordered circle in figure for that calculation of $w$ and $b$ is necessary. In test phase, the class of new data vector $x$ can be predict by projecting $\mathrm{x}$ on weight vector $\mathrm{w}$,

$$
\mathrm{F}(\mathrm{x})=\mathrm{w} \cdot \mathrm{x}+\mathrm{b}
$$

SVM optimization problem,

$$
\begin{array}{cl} 
& \min \frac{1}{2}\|w\|^{2}+C \sum_{i} \xi_{i} \\
\text { s.t. } & y_{i}\left(w x_{i}+b\right) \geq 1-\xi_{i}, \xi_{i}>0 \forall i .
\end{array}
$$

The regularization parameter $\mathrm{C}$ has higher value which corresponds to stronger penalties. To solve this problem, it is possible to rewrite it in terms of the positive Lagrangian multipliers $\alpha_{\mathrm{i}}$. Then, the dual representation requires maximizing

$$
L_{D}=\sum_{i=1}^{l} \alpha_{i}-\frac{1}{2} \sum_{i, j=1}^{l} \alpha_{i} \alpha_{j} y_{i} y_{j}\left(x_{i} \cdot x_{j}\right)
$$

$$
\begin{gathered}
\text { subject to } 0 \leq \alpha_{i} \leq \mathrm{C} \text { and } \sum_{i} \alpha_{i} y_{i}=0 \text { and yields } \\
w=\sum_{i}^{N_{s}} y_{i} \alpha_{i} x_{i} .
\end{gathered}
$$

The SVM classifier is controlled by the regularization parameter $\mathrm{C}$ and the bandwidth.

\section{Performance measures}

To examine performance of the classification algorithm we used f1-score technique. F1-score is calculated using following equation:

$$
\begin{gathered}
\text { Precision }=\frac{T P}{T P+F P} \\
\text { Recall }=\frac{T P}{T P+F N} \\
\text { F1 Score }=2 * \frac{\text { Precision } * \text { Recall }}{\text { Precision }+ \text { Recall }}
\end{gathered}
$$




\section{A Comparative Study of Classification Techniques for P300 Speller}

where, $\mathrm{TP}=$ True Positive; $\mathrm{TN}=$ True Negative;

$\mathrm{FP}=$ False Positive;FN=False Negative.

\section{IMPLEMENTATION}

Following fig 6 illustrated flow chart of the P300 speller system. Feature Extraction perform on EEG data to extract useful features of P300 signals. Classification algorithms are applied on feature vector which result in P300 and non-P300 class [19]. Which is used to predict character to right spell. We can measure performance of the classification algorithms.

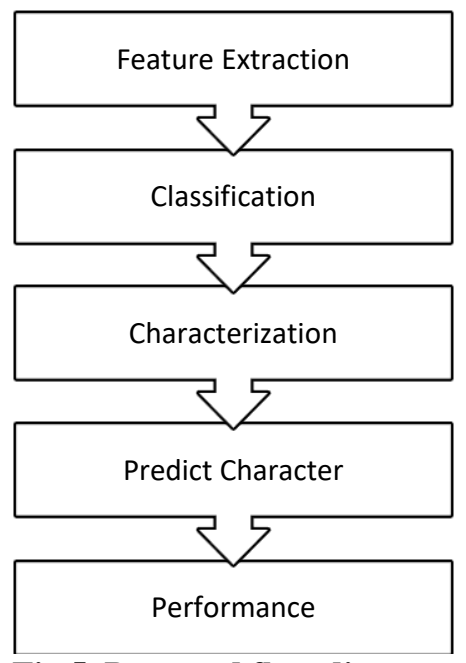

Fig 5. Proposed flow diagram

P300 speller implemented in Python because it has become an important platform for scientific computing. Open source libraries like NumPy, SciPy, Matplotlib, IPython are used for scientific computation, Scikitlearn is used for machine learning techniques and Pandas is used for data analysis. Python is platform independent which makes it attractive for research institutions.

Wyrm, an open source BCI toolbox in Python. To load the BCI competition III dataset II and to perform some preprocessing task we used Wyrm toolbox. We are using some key features of the Wyrm like design data structure, demonstrate and validate result.

\section{A. Data structure}

In BCI, EEG data was gathered from a 64-channel headset cap as shown in Figure 4.4. The diagram shows 64electrode position and channel numbers.

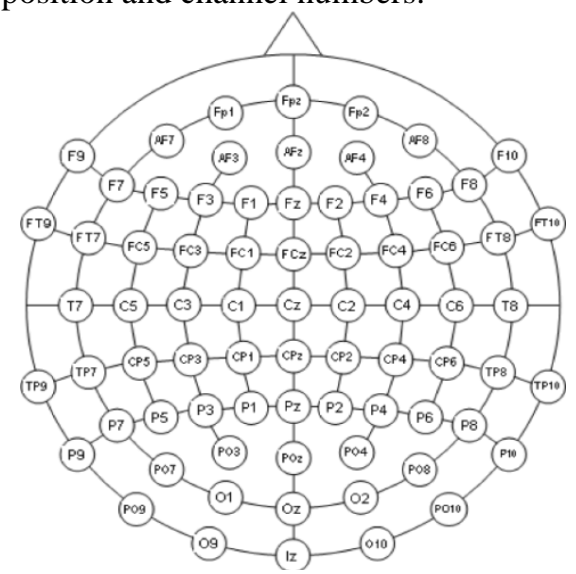

Fig 6. 64 channel electrode position and its number [5]
BCI competition III dataset II $[14,15]$ represents a record of P300 evoked potentials recorded with BCI2000 software described by Farwell and Donchin [20]. The dataset consists of 2 subject recorded P300 data.

\section{B. Result and Analysis}

Fig 7 illustrated the plot of Signed $r^{2}$ values to extract feature. The channels are listed from frontal to occipital and within each row from left to right. The blobs in fig shows the time intervals for each channel.

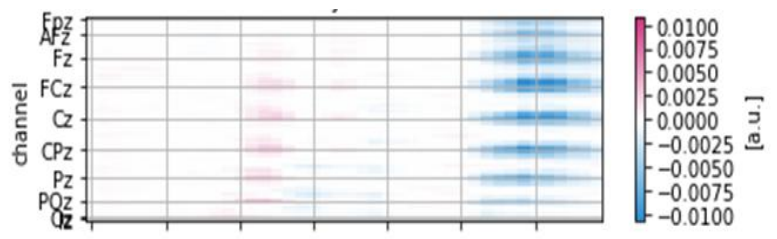

Fig 7. Plot of Signed $\mathbf{r}^{2}$-values

Fig 8 illustrated target and non-target class wise signals for selected channels are $\mathrm{Fz}, \mathrm{Cz}$ and $\mathrm{Pz}$
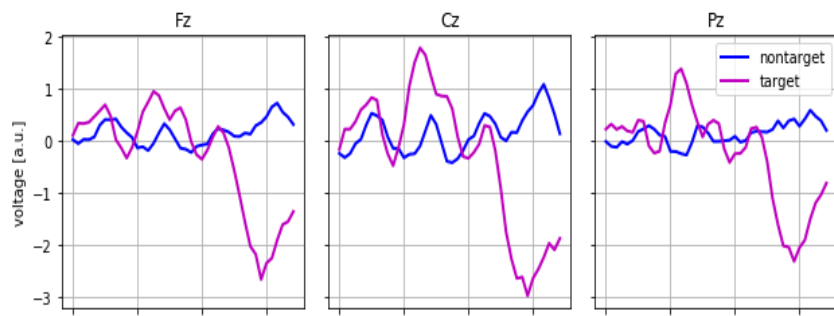

Fig 8. Class wise signal for 3 selected channels $(F z$, Cz\&Pz)

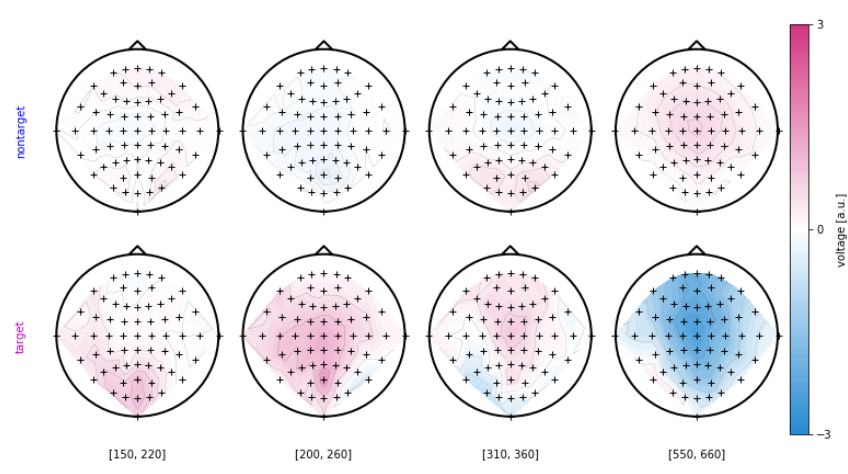

Fig 9. Spatial topographies of average voltage distribution for the different time interval

Fig 9 shows the spatial topographies of the average voltage distribution for the different time intervals used for classification. LDA and SVM classification algorithms are used to classify. LDA and SVM both have $91.0 \%$ classification accuracy. Using classified signals, we constructed labels and compared it with the true label as shown below.

Constructed labels:

WQXPLZCOMRKOW7YFZDEZ1DPI9NN2GRKDJCUJRMEUOCOJD2UFYPOO6 J7LDGYEGOA5VHNEKBW4OO1TDOILUEE5BFAEEXAW_K3R3MRU

True labels:

WQXPLZCOMRKO97YFZDEZ1DPI9NNVGRQDJCUVRMEUOOOJD2UFYPOO6 J7LDGYEGOA5VHNEHBTXOO1TDOILUEE5BFAEEXAW K4R3MRU 


\section{A Comparative Study of Classification Techniques for P300 Speller}

\section{CONCLUSION}

The proposed system of P300 speller can reduce calibration time to classify target and non-target signals and improve information transfer rate. In our system, we used LDA and SVM classification algorithm. Both the classifier got $91.0 \%$ classification accuracy. Higher the classification accuracy gave us better characterization result.

\section{FUTURE WORK}

In future, we can apply different machine learningalgorithms to reduce calibration time and improveperformance of classification so that accuracy ofcharacterization will increase.

\section{REFERENCES}

1. Abdulkader, Sarah N., AymanAtia, and Mostafa-Sami M. Mostafa. "Brain computer interfacing: Applications and challenges." Egyptian Informatics Journal 16.2 (2015): 213-230.

2. Guy, Violaine, et al. "Brain computer interface with the P300 speller: Usability for disabled people with amyotrophic lateral sclerosis." Annals of physical and rehabilitation medicine 61.1 (2018): 5-11.

3. Krigolson, Olave E., et al. "Choosing MUSE: Validation of a lowcost, portable EEG system for ERP research." Frontiers in neuroscience 11 (2017): 109.

4. Renard, Yann, et al. "Openvibe: An open-source software platform to design, test, and use brain-computer interfaces in real and virtual environments." Presence: teleoperators and virtual environments 19.1 (2010): 35-53

5. Blankertz, B. "Documentation second wadsworth BCI dataset (P300 evoked potentials) data acquired using BCI2000 P300 Speller Paradigm." BCI Classification Contest November (2002).

6. Schalk, Gerwin, et al. "BCI2000: a general-purpose brain-computer interface (BCI) system." IEEE Transactions on biomedical engineering 51.6 (2004): 1034-1043.

7. Venthur, Bastian, and Benjamin Blankertz. "Mushu, a free-and open source BCI signal acquisition, written in python." 2012 Annual International Conference of the IEEE Engineering in Medicine and Biology Society. IEEE, 2012.

8. Venthur, Bastian, et al. "Wyrm: A brain-computer interface toolbox in python." Neuroinformatics 13.4 (2015): 471-486.

9. Venthur, Bastian, et al. "Pyff---A Pythonic Framework for Feedback Applications and Stimulus Presentation in Neuroscience." Frontiers in neuroscience 4 (2010): 179

10. Hongchang Shan, Yu Liu, TodorStefanov "A Simple Convolutional Neural Network for Accurate P300 Detection and Character Spelling in Brain Computer Interface" Artificial Intelligence (IJCAI-18).

11. Qi, Hongzhi, et al. "A Speedy Calibration Method Using Riemannian Geometry Measurement and Other-Subject Samples on A P300 Speller." IEEE Transactions on Neural Systems and Rehabilitation Engineering 26.3 (2018): 602-608

12. Elsawy, Amr S., et al. "A principal component analysis ensemble classifier for P300 speller applications." Image and Signal Processing and Analysis (ISPA), 2013 8th International Symposium on. IEEE, 2013.

13. Hoffmann, Ulrich, Jean-Marc Vesin, and TouradjEbrahimi. "Recent advances in brain-computer interfaces." IEEE International Workshop on Multimedia Signal Processing (MMSP07). No. LTS-CONF-2007063. 2007.

14. Kaper, Matthias, et al. "BCI competition 2003-data set Ilb: support vector machines for the P300 speller paradigm." IEEE Transactions on Biomedical Engineering 51.6 (2004): 1073-1076.

15. Rakotomamonjy, Alain, and Vincent Guigue. "BCI competition III: dataset II-ensemble of SVMs for BCI P300 speller." IEEE transactions on biomedical engineering 55.3 (2008): 1147-1154

16. $\mathrm{Xu}, \mathrm{Tao}$, et al. "Learning Emotions EEG-based Recognition and Brain Activity: A Survey Study on BCI for Intelligent Tutoring System." Procedia Computer Science 130 (2018): 376-382.

17. $\mathrm{Li}, \mathrm{Qi}$, et al. "Training set extension for SVM ensemble in P300speller with familiar face paradigm." Technology and Health Care Preprint (2018): 1-14.

18. Fabien Lotte. "A Tutorial on EEG Signal Processing Techniques for Mental State Recognition in Brain-Computer Interfaces." Eduardo Reck Miranda; Julien Castet. Guide to Brain-Computer Music Interfacing, Springer, 2014
19. Qu, Jun, et al. "A Novel Three-Dimensional P300 Speller Based on Stereo Visual Stimuli." IEEE Transactions on Human-Machine Systems (2018).

20. L. A. Farwell e E. Donchin, "Talking off the top of your head: toward a mental prosthesis utilizing eventrelated brain potentials", Electroencephalogr. Clin. Neurophysiol., vol. 70, n. 6, pagg. 510-523, 1988.

21. Lee, Yu-Ri, and Hyoung-Nam Kim. "A data partitioning method for increasing ensemble diversity of an eSVM-based P300 speller." Biomedical Signal Processing and Control 39 (2018): 53-63.

22. Liu, Mingfei, et al. "Deep learning based on Batch Normalization for P300 signal detection." Neurocomputing 275 (2018): 288-297.

23. Zhimin Lin1, Chi Zhang 1, Ying Zeng2,1, Li Tong1 \& Bin Yan1 "A novel P300 BCI speller based on the Triple RSVP paradigm" Scientific reports 8.1 (2018) 